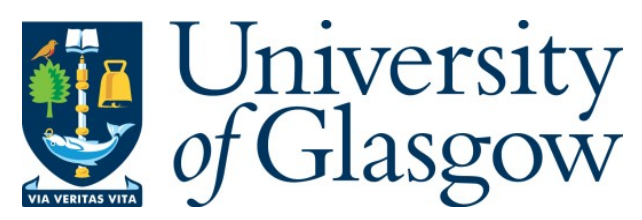

Grant, J., McCrindle, I.J.H., Li, C., and Cumming, D.R.S. (2014)

Multispectral metamaterial absorber. Optics Letters, 39 (5). pp. 1227-1230. ISSN 0146-9592

Copyright $@ 2014$ The Authors

http://eprints.gla.ac.uk/92955

Deposited on: 23 April 2014

Enlighten - Research publications by members of the University of Glasgow http://eprints.gla.ac.uk 


\title{
Multispectral metamaterial absorber
}

\author{
J. Grant, ${ }^{*}$ I. J. H. McCrindle, C. Li, and D. R. S. Cumming \\ School of Engineering, University of Glasgow, Glasgow G12 8LT, UK \\ ${ }^{*}$ Corresponding author: james.grant@glasgow.ac.uk \\ Received November 26, 2013; accepted January 8, 2014; \\ posted January 24, 2014 (Doc. ID 201834); published February 24, 2014
}

\begin{abstract}
We present the simulation, implementation, and measurement of a multispectral metamaterial absorber (MSMMA) and show that we can realize a simple absorber structure that operates in the mid-IR and terahertz (THz) bands. By embedding an IR metamaterial absorber layer into a standard THz metamaterial absorber stack, a narrowband resonance is induced at a wavelength of $4.3 \mu \mathrm{m}$. This resonance is in addition to the $\mathrm{THz}$ metamaterial absorption resonance at $109 \mu \mathrm{m}(2.75 \mathrm{THz})$. We demonstrate the inherent scalability and versatility of our MSMMA by describing a second device whereby the MM-induced IR absorption peak frequency is tuned by varying the IR absorber geometry. Such a MSMMA could be coupled with a suitable sensor and formed into a focal plane array, enabling multispectral imaging. (C) 2014 Optical Society of America

OCIS codes: (160.3918) Metamaterials; (040.2235) Far infrared or terahertz; (310.6628) Subwavelength structures, nanostructures.

http://dx.doi.org/10.1364/OL.39.001227
\end{abstract}

Metamaterials (MMs) are arrays of subwavelength elements, the structure of which, rather than their composition, dictates their electromagnetic properties. Initial interest in MMs arose due to their ability to exhibit exotic electromagnetic effects impossible to achieve with natural materials. Since the first theoretical [1] and experimental demonstration [2] of their unique properties, research into the topic has grown rapidly. MMs can provide a highly controllable electromagnetic response in different frequency bands, and to date MMs have been demonstrated in every technologically relevant spectral range, enabling investigations into new possibilities including perfect lenses [3], hyperspectral lenses [4], and invisibility cloaks [5]. A burgeoning branch of MMs currently provoking wide interest is the topic of so-called MM-perfect absorbers. By manipulating the effective electrical permittivity, $\varepsilon$, and magnetic permeability, $\mu$, absorption close to unity is possible [6]. Traditional electromagnetic absorber structures such as Jaumann absorbers or Salisbury screens [7] require a thickness of $\lambda / 4$, where $\lambda$ is the center wavelength of the incident electromagnetic radiation. MM absorbers, on the other hand, can absorb the incident radiation in far thinner layers, typically $\lambda / 35$ [8]. The motivation for studying MM absorbers lies in their potential applications as selective thermal emitters [9], spatial light modulators [10] and in detection and sensing [11]. Single-band, dual-band and broadband MM absorbers have been demonstrated across all the major wavebands, including the microwave [], THz [ㅁ, 12-14], IR [15], and visible [16]. An extensive overview of the topic can be found in [17]. One application where a thin absorption layer is desirable is bolometric detection, whereby incident electromagnetic radiation is absorbed by a material and then sensed by a high temperature coefficient resistor. The periodic nature of MM absorbers lends itself to coupling with microbolometer sensing elements to form a focal plane array (FPA). Such an $11 \times 11$ MM-based FPA operating in the $\mathrm{S}$ band (2-4 GHz) has been presented [18] whereby the associated read-out electronics are discrete and implemented on a printed circuit board. At THz frequencies, where the MM array dimensions are smaller, the potential of MM absorbers for $\mathrm{THz}$ imaging applications has been demonstrated via the monolithic integration of MM absorbers into a standard foundry CMOS process and subsequent post deposition of vanadium oxide microbolometer sensor elements [19].

To date, many authors have demonstrated multispectral MM absorption [ $\underline{13}, \underline{20}]$, but the multiple absorption bands occur in only one region of the electromagnetic spectrum. In contrast, the MSMMA presented here has absorption bands in distinct regions of the electromagnetic spectrum, namely the mid-IR $(\sim 4 \mu \mathrm{m})$ and $\mathrm{THz}$ (2.9 THz). True multispectral filtering of visible and $\mathrm{THz}$ radiation from a single thin film has been demonstrated by hybridizing optical plasmonics and a metamaterial filter [21]. In addition, a device capable of filtering visible radiation and $\mathrm{THz}$ absorption has been presented by McCrindle et al. [22]. Integrating a microbolometer sensor with our MSMMA would result in a coaxial THz and IR sensor that maximizes the spectral information acquired per unit area and could be scaled to FPA formats to yield a $\mathrm{THz}$ and IR image.

The schematic of a single unit cell of our MSMMA is shown in Fig. 1(a). It consists of a typical THz single-band MM absorber, based on the design presented in [12], into which is embedded an array of metallic crosses that, along with a continuous ground plane, form the IR absorber. In each single THz unit cell, there are 15 subunit IR cells. The unit cell period is $30 \mu \mathrm{m}$, while the subunit cell period is $2 \mu \mathrm{m}$. The metallic cross-shaped structures are examples of an electric ring resonator (ERR) and couple strongly to uniform electric fields, but negligibly to a magnetic field. By pairing the ERR with a ground plane, the magnetic component of the incident wave couples to the center section of the ERR and the ground plane, thus generating antiparallel currents resulting in a resonant response. The ERR determines the electric response, while the insulator type and thickness between the ERR and the ground plane determines the magnetic response. In practical terms, the cross-arm length dominates the resonance frequency peak position, while the insulating layer thickness and refractive index properties dictate the absorption magnitude. The optimum insulator layer thickness for attaining high absorption between the ERR and the ground plane scales with 
(a)
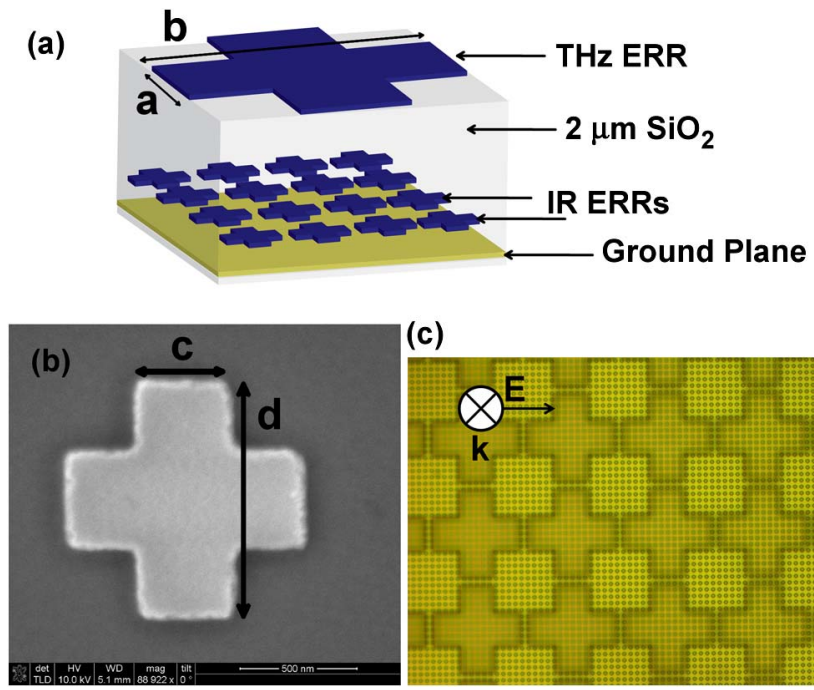

(c)

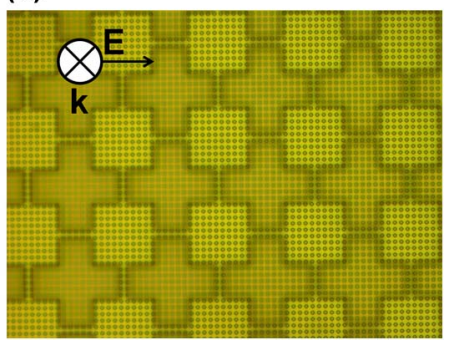

Fig. 1. (a) 3D schematic of the MSMMA (not to scale), (b) scanning electron microscope image of a single IR ERR, and (c) optical microscope image of the MSMMA.

frequency. In our MSMMA, the $\mathrm{SiO}_{2}$ thickness between the IR ERR and the ground plane is $50 \mathrm{~nm}$ and between the THz ERR and ground plane is $2.05 \mu \mathrm{m}$. The MSMMA geometric parameters labeled in Figs. 1(a) and 1(b) are as follows: $a=10 \mu \mathrm{m}, b=27 \mu \mathrm{m}, \quad c=0.4 \mu \mathrm{m}$, and $d=0.8 \mu \mathrm{m}$.

Before committing to fabrication, extensive finitedifference time-domain (FDTD) simulations were performed to determine the optimum geometry for the MSMMA. A 3D simulation model was used throughout to investigate the behavior of the MSMMA in the midIR $(3-10 \mu \mathrm{m})$ and terahertz $(1.5-4 \mathrm{THz})$ wavelength regimes. Our 3D simulations were performed with a plane wave source incident in the $z$ direction on the multilayer unit cell and with perfectly matched layer boundary conditions. Taking advantage of the inherent symmetry of the structure and to reduce computation demands, antisymmetric and symmetric boundary conditions were used in the $x$ and $y$ directions, respectively. A uniform mesh was used in the region around the metal with maximum mesh cell sizes of $\Delta x=\Delta y=\Delta z=5 \mathrm{~nm}$. In the IR region, the metallic regions (ERRs and ground plane) were modeled using the default Au Palik refractive index data available in the Lumerical material database [23-25]. In the $\mathrm{THz}$ range, no Palik data was available; instead a frequency independent conductivity of $4 \times 10^{7} \mathrm{Sm}^{-1}$ was used. For both the IR and $\mathrm{THz}$ regions Palik data was used for the $\mathrm{SiO}_{2}$ insulating layer [23-25]. Reflection and transmission spectra were recorded at planes $100 \mu \mathrm{m}$ above and $100 \mu \mathrm{m}$ below the top THz ERR.

Once the optimum MSMMA design had been established, standard metal evaporation, plasma deposition, and e-beam lithography techniques were used to fabricate devices. First a 20/130 nm Ti/Au metallic ground plane was evaporated onto a $15 \mathrm{~mm} \times 15 \mathrm{~mm}$ silicon substrate. A $50 \mathrm{~nm}$ thick layer of $\mathrm{SiO}_{2}$ was then deposited by plasma-enhanced chemical vapor deposition (PECVD). The IR ERR layer, spanning an area of $12 \mathrm{~mm} \times 12 \mathrm{~mm}$, was defined in a bi-layer of PMMA e-beam resist using a Vistec VB6 e-beam tool and a 20/130 nm film of Ti/Au evaporated and lifted off in hot acetone. A further $2 \mu \mathrm{m}$ of PECVD $\mathrm{SiO}_{2}$ was deposited on top of the IR ERR layer and finally the THz ERR layer was again defined using electron-beam lithography in a bi-layer of PMMA, a 20/130 nm Ti/Au layer evaporated and lifted off in hot acetone. A scanning electron microscope (SEM) image of a single IR ERR is shown in Fig. 1(b), while an optical image of the fully fabricated MSMMA is shown in Fig. 1(c).

Samples were characterized under vacuum in a Bruker IFS 66v/S Fourier transform infrared spectrometer in reflection mode at $30^{\circ}$ incidence. For the IR region, the reflection and transmission measurements were performed using a $\mathrm{SiC}$ globar source, $\mathrm{KBr}$ beam splitter, and a deuterated, L-alanine-doped triglycine sulfate (DLaTGS) pyroelectric detector. THz measurements required the use of a $\mathrm{Hg}$ source, a Mylar beam splitter, and a DLaTGS detector with a polyethylene window. The measured reflection spectra were normalized to that of a gold mirror. Since the Ti/Au ground plane thickness is greater than the skin depth at $\mathrm{THz}$ frequencies and above $(1 \mathrm{THz} \approx 75 \mathrm{~nm})$, there is negligible transmission through the MSMMA, therefore, the absorption characteristics can be determined directly from the reflection measurements. The resulting absorption, $A$, was therefore calculated using $A(\omega)=1-R(\omega)$, where $R$ is the reflection.

Figure 2(a) shows FDTD simulation and experimental data for a standalone MM IR absorber consisting of a ground plane, $50 \mathrm{~nm} \mathrm{SiO}_{2}$ layer, and metallic IR ERRs. The geometric dimensions of the IR ERRs are identical to that used in the MSMMA. The experimental spectrum has an absorption peak of $83 \%$ magnitude at a wavelength of $4.09 \mu \mathrm{m}$. In comparison, the simulated spectrum yields an absorption magnitude of $98 \%$ at $3.89 \mu \mathrm{m}$, implying good agreement between the simulation and experimental data. Also shown in Fig. 2(a) is the IR spectral response of a standalone $\mathrm{THz}$ absorber. There are a series of small absorption peaks below $5 \mu \mathrm{m}$ and a large, more prominent, broad, absorption peak present at $7.7 \mu \mathrm{m}$. The absorption mechanism for the peak at $7.7 \mu \mathrm{m}$ is attributed to classical dielectric loss, as it is well known that the absorption coefficient of $\mathrm{SiO}_{2}$ increases markedly from 6 to $8 \mu \mathrm{m}$ [23-25]. Significantly, there is only $20 \%$ absorption at $4.09 \mu \mathrm{m}$. The experimental absorption spectrum of the MSMMA, shown in Fig. 2(a), has two major peaks. In addition to the peak at $7.7 \mu \mathrm{m}$, there is a second peak with a maximum absorption magnitude of $47 \%$ at $4.3 \mu \mathrm{m}$. Again, this experimental data compares favorably with the simulated characteristics that exhibit a major absorption peak at $4.63 \mu \mathrm{m}$ of $49 \%$ magnitude and confirms that this peak is a direct consequence of the IR MM absorber layer embedded in the MSMMA.

For the 80-200 $\mu \mathrm{m}$ (i.e., THz) range, the experimental absorption spectrum of the MSMMA is shown in Fig. 2(b). An absorption magnitude of $77 \%$ is attained at the resonance wavelength of $109 \mu \mathrm{m}$. As evident from Fig. 2(b), good agreement is obtained between the experimental and simulated absorption spectra. There is also little difference in the experimental absorption characteristics of the standalone $\mathrm{THz} \mathrm{MM}$ absorber and the MSMAA. Figure $2(\mathrm{c})$ shows the absorption spectrum 

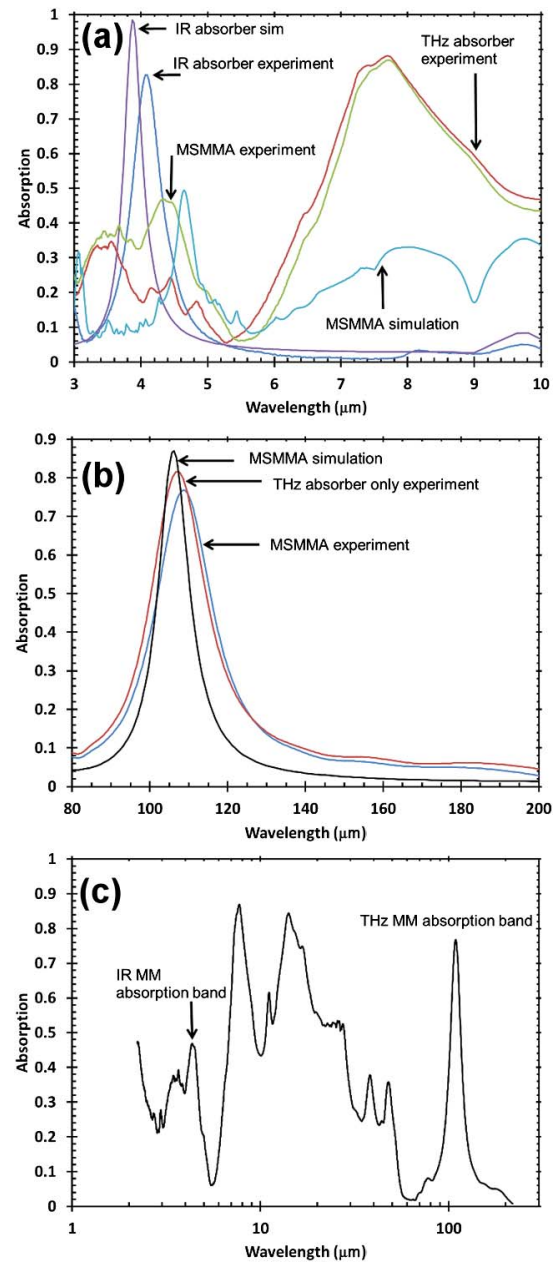

Fig. 2. (a) Experimental and simulated FDTD data of a standalone IR absorber, THz absorber, and MSMMA in the IR region (3-10 $\mu \mathrm{m})$, (b) experimental and simulated FDTD spectral characteristics of a stand-alone THz absorber and MSMMA in the terahertz region $(80-200 \mu \mathrm{m})$, and (c) experimental spectral absorption of the MSMMA from 2.2 to $212 \mu \mathrm{m}$ (136-1.4 THz).

for the MSMMA from 2.2 to $212 \mu \mathrm{m}$ and reveals numerous absorption peaks. The two peaks attributed to MM-based absorption are labeled accordingly.

To confirm the origin of the three major absorption peaks associated with the MSMMA, we simulated the absorption distribution in the $x z$ plane using Lumerical FDTD. Figure 3 shows the simulated absorption distribution in the $x z$ plane for the three major absorption peaks of the MSMMA. To aid the understanding of the absorption distribution for each absorption peak, the $x z$ plot of the refractive index is shown in Fig. 3(a). The red areas denote $\mathrm{Au}$, the green areas are $\mathrm{SiO}_{2}$, and the blue area is air. The top of the ground plane is at $z=0.2 \mu \mathrm{m}$, the bottom of the IR ERR is at $z=0.25 \mu \mathrm{m}$, and the bottom of the THz ERR layer is at $z=2.25 \mu \mathrm{m}$. For the absorption peak at $4.64 \mu \mathrm{m}$, the energy is mainly absorbed in the metal and insulating layers that comprise the IR MM absorber. In contrast, at $7.7 \mu \mathrm{m}$, the majority of the energy is absorbed in the first $800 \mathrm{~nm}$ of the $\mathrm{SiO}_{2}$ beneath the THz ERR, and there is negligible contribution from the IR MM asborber structure. As can be clearly seen in Fig. 3(c), at $109 \mu \mathrm{m}$, the absorption occurs in the
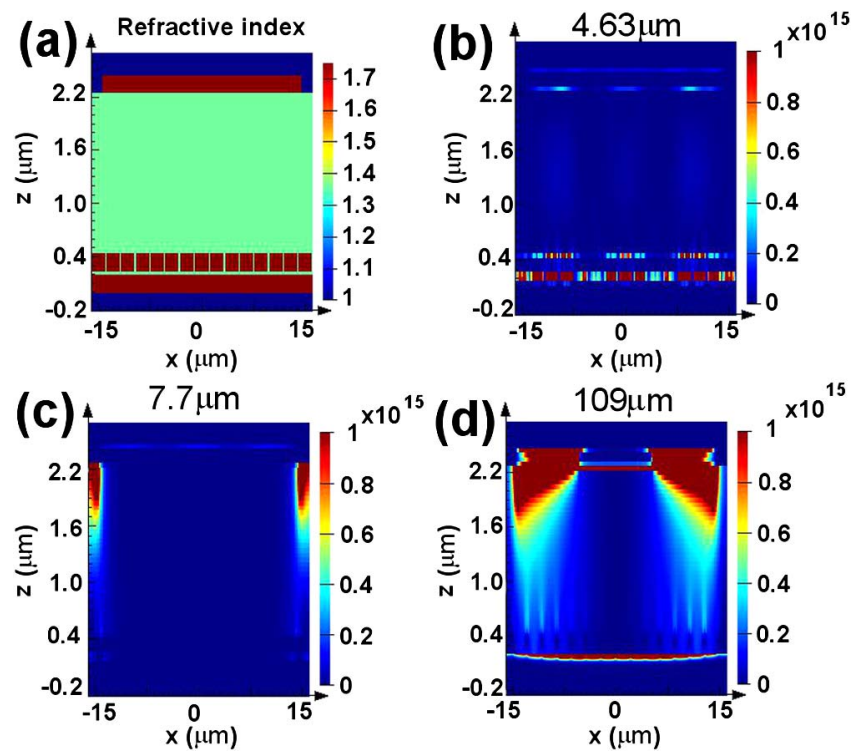

Fig. 3. (a) Refractive index plot in the $x z$ plane of the MSMMA and simulated FDTD absorption distribution in the $x z$ plane at the three major absorption peaks of (b) $4.63 \mu \mathrm{m}$, (c) $7.7 \mu \mathrm{m}$, and (d) $109 \mu \mathrm{m}$.

THz ERR metallic layer and first few hundred micrometers of $\mathrm{SiO}_{2}$, supporting our hypothesis that the THz MM absorber is responsible for this absorption band.

Numerous possibilities exist with our MSMMA to tune the absorption bands to any desired frequency. As a demonstration, we have simulated, fabricated, and characterized a second MSMMA, hereafter known as MSMMA2. This device is constructed with the same layer thicknesses and materials as the MSMMA with two exceptions to the IR MM absorber component: (1) the IR ERR crossarm length is $1.6 \mu \mathrm{m}$ as opposed to $0.8 \mu \mathrm{m}$ and (2) the $\mathrm{SiO}_{2}$ thickness between the ground plane and the IR ERRs is $100 \mathrm{~nm}$ instead of $50 \mathrm{~nm}$. It is well known that increasing the cross-arm length and fixing the period of the structure results in a red shift of the resonance frequency, while in order to attain high absorption, the insulating layer thickness between the ground plane and ERR layer must increase with increasing wavelength. The spectral absorption properties of the standalone IR absorber, labeled as IR2, are shown in Fig. 4(a). An absorption magnitude of $75 \%$ is obtained at $6.1 \mu \mathrm{m}$. For the combined IR and THz MM absorber device, MSMMA2, the IR absorption peak magnitude drops to $52 \%$, and the frequency peak position red shifts to $6.28 \mu \mathrm{m}$. The full absorption spectrum from 2.2 to $240 \mu \mathrm{m}$ can be seen in Fig. 4(b). Modifying the IR ERR structure has no effect on the absorption magnitude (77\%) and frequency peak position $(112 \mu \mathrm{m})$ of the THz MM absorber compared to the MSMMA device.

In summary, we have simulated, fabricated, and characterized a multispectral metamaterial absorber. The MSMMA has experimental absorption peaks at 4.3 and $112 \mu \mathrm{m}$ with magnitude of $47 \%$ and $77 \%$, respectively, and the results are corroborated via FDTD simulations. A natural application for this device is multispectral imaging. The periodic nature of MM structures lends itself to forming arrays. If a sensor, such as a microbolometer, were to be coupled with the MSMMA then a 

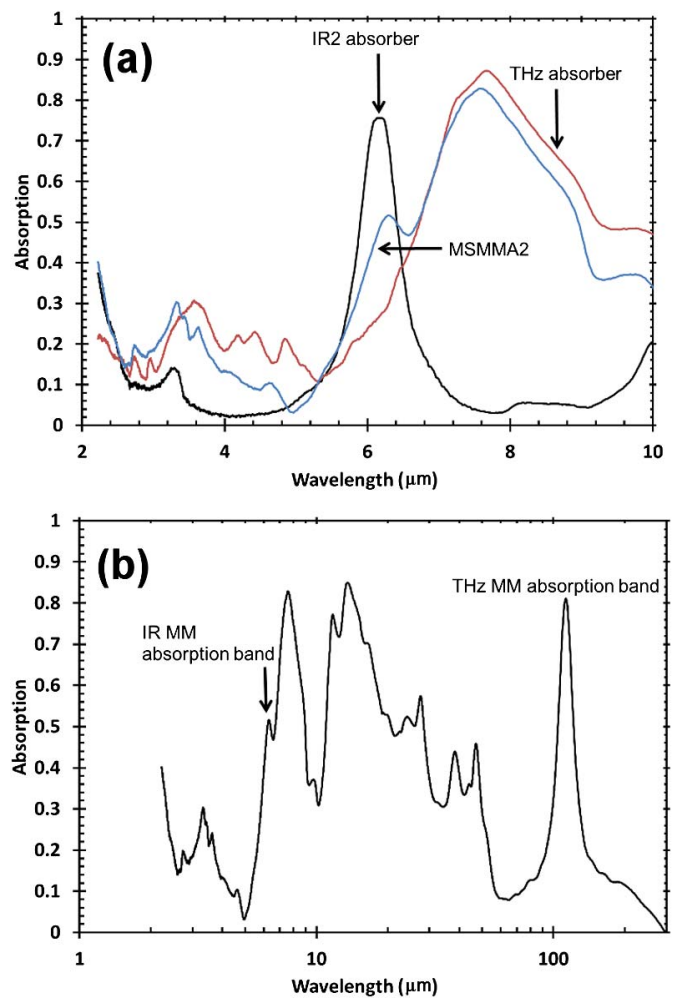

Fig. 4. (a) Experimental spectral absorption characteristics of the IR2 absorber and MSMMA2 absorber in the $2-10 \mu \mathrm{m}$ wavelength range and (b) absorption spectrum of the MSMMA2 from 2.2 to $240 \mu \mathrm{m}$.

multiwavelength focal plane array could be realized. Such an imaging device could render images at two distinct wavelengths in the IR and THz, e.g., at 4.3 and $112 \mu \mathrm{m}$. Image fusion, the process of combining relevant information from two or more images into a single image, would render a resulting image that is more informative and data rich than either of the individual input images.

The authors would like to thank the staff of the James Watt Nanofabrication Centre at the University of Glasgow for help in fabricating the devices reported in this Letter. This research was funded from EPSRC grants EP/I017461/1 and EP/J018678/1.

\section{References}

1. J. B. Pendry, A. J. Holden, D. J. Robbins, and W. J. Stewart, IEEE Trans. Microwave Theor. Tech. 47, 2075 (1999).
2. D. R. Smith, W. J. Padilla, D. C. Vier, S. C. Nemat-Nasser, and S. Schultz, Phys. Rev. Lett. 84, 4184 (2000).

3. N. Fang and X. Zhang, Appl. Phys. Lett. 82, 161 (2003).

4. K. Aydin, I. Bulu, and E. Ozbay, Appl. Phys. Lett. 90, 254102 (2007).

5. D. Schurig, J. J. Mock, B. J. Justice, S. A. Cummer, J. B. Pendry, A. F. Starr, and D. R. Smith, Science 314, 977 (2006).

6. N. I. Landy, C. M. Bingham, T. Tyler, N. Jokerst, D. R. Smith, and W. J. Padilla, Phys. Rev. B 79, 125104 (2009).

7. B. A. Munk, Frequency Selective Surfaces (Wiley, 2000).

8. N. I. Landy, S. Sajuyigbe, J. J. Mock, D. R. Smith, and W. J. Padilla, Phys. Rev. Lett. 100, 207402 (2008).

9. X. L. Liu, T. Tyler, T. Starr, A. F. Starr, N. M. Jokerst, and W. J. Padilla, Phys. Rev. Lett. 107, 045901 (2011).

10. D. Shrekenhamer, C. M. Watts, and W. J. Padilla, Opt. Express 21, 12507 (2013).

11. H. Tao, E. A. Kadlec, A. C. Strikwerda, K. B. Fan, W. J. Padilla, R. D. Averitt, E. A. Shaner, and X. Zhang, Opt Express 19, 21620 (2011).

12. J. Grant, Y. Ma, S. Saha, L. B. Lok, A. Khalid, and D. R. S. Cumming, Opt. Lett. 36, 1524 (2011).

13. Y. Ma, Q. Chen, J. Grant, S. C. Saha, A. Khalid, and D. R. S. Cumming, Opt. Lett. 36, 945 (2011).

14. J. Grant, Y. Ma, S. Saha, A. Khalid, and D. R. S. Cumming, Opt. Lett. 36, 3476 (2011).

15. X. L. Liu, T. Starr, A. F. Starr, and W. J. Padilla, Phys. Rev. Lett. 104, 207403 (2010).

16. W. R. Zhu, X. P. Zhao, B. Y. Gong, L. H. Liu, and B. Su, Appl. Phys. A 102, 147 (2011).

17. C. M. Watts, X. L. Liu, and W. J. Padilla, Adv. Mater. 24, Op98 (2012).

18. D. Shrekenhamer, W. Xu, S. Venkatesh, D. Schurig, S. Sonkusale, and W. J. Padilla, Phys. Rev. Lett. 109, 177401 (2012).

19. J. Grant, I. Escorcia-Carranza, C. Li, I. J. H. McCrindle, J. Gough, and D. R. S. Cumming, Laser Photon. Rev. 7, 1043 (2013).

20. X. P. Shen, T. J. Cui, J. M. Zhao, H. F. Ma, W. X. Jiang, and H. Li, Opt. Express 19, 9401 (2011).

21. I. J. H. McCrindle, J. Grant, T. D. Drysdale, and D. R. S. Cumming, Opt. Express 21, 19142 (2013).

22. I. J. H. McCrindle, J. Grant, T. D. Drysdale, and D. R. S. Cumming, "Multi-spectral materials: hybridisation of optical plasmonic filters and a terahertz metamaterial absorber," Adv. Opt. Mater., doi: 10.1002/adom.201300408 (to be published).

23. E. D. Palik, Handbook of Optical Constants of Solids (Elsevier, 1985), Vol. I.

24. E. D. Palik, Handbook of Optical Constants of Solids (Elsevier, 1985), Vol. II.

25. E. D. Palik, Handbook of Optical Constants of Solids (Elsevier, 1985), Vol. III. 